\title{
Yang-Mills instantons on cones and sine-cones over nearly Kähler manifolds
}

\author{
Karl-Philip Gemmer, ${ }^{a}$ Olaf Lechtenfeld, ${ }^{a, b}$ Christoph Nölle ${ }^{a}$ and Alexander D. Popov ${ }^{c}$ \\ ${ }^{a}$ Institut für Theoretische Physik, Leibniz Universität Hannover, \\ Appelstraße 2, 30167 Hannover, Germany \\ ${ }^{b}$ Centre for Quantum Engineering and Space-Time Research, Leibniz Universität Hannover, \\ Welfengarten 1, 30167 Hannover, Germany \\ ${ }^{c}$ Bogoliubov Laboratory of Theoretical Physics, JINR, \\ 141980 Dubna, Moscow Region, Russia \\ E-mail: Karl-Philip.Gemmer@itp.uni-hannover.de, \\ Olaf .Lechtenfeld@itp.uni-hannover.de, \\ Christoph. Noelle@itp.uni-hannover.de, popov@theor.jinr.ru
}

ABSTRACT: We present a unified eight-dimensional approach to instanton equations on several seven-dimensional manifolds associated to a six-dimensional homogeneous nearly Kähler manifold. The cone over the sine-cone on a nearly Kähler manifold has holonomy group $\operatorname{Spin}(7)$ and can be foliated by submanifolds with either holonomy group $G_{2}$, a nearly parallel $G_{2}$-structure or a cocalibrated $G_{2}$-structure. We show that there is a $G_{2}$-instanton on each of these seven-dimensional manifolds which gives rise to a $\operatorname{Spin}(7)$-instanton in eight dimensions. The well-known octonionic instantons on $\mathbb{R}^{7}$ and $\mathbb{R}^{8}$ are contained in our construction as the special cases of an instanton on the cone and on the cone over the sine-cone, both over the six-sphere, respectively.

KEYwords: Flux compactifications, Solitons Monopoles and Instantons, Differential and Algebraic Geometry

ARXIV EPRINT: 1108.3951 


\section{Contents}

1 Introduction 1

2 Cones, sine-cones and cylinders over nearly Kähler manifolds 3

2.1 Nearly Kähler cosets $G / H \quad 4$

$\begin{array}{lll}2.2 & G_{2} \text {-structures } & 7\end{array}$

2.3 Spin(7)-holonomy from $G_{2}$-structure manifolds 8

3 Instantons and submanifolds $\quad 8$

4 Yang-Mills actions and Chern-Simons flows 11

5 Explicit ansätze for the connection $\quad \mathbf{1 2}$

5.1 Reduced instanton equations for gauge group $G \quad 13$

$\begin{array}{ll}5.2 & \text { Solutions for gauge group } G \\ 5.3 & 16\end{array}$

$\begin{array}{ll}5.3 \text { Gauge groups } G_{2} \text { and } \operatorname{Spin}(7) & 17\end{array}$

$\begin{array}{llr}6 & \text { Conclusions and outlook } & 19\end{array}$

A Comparing the instanton on $\mathbb{R}^{7}$ and on the cone over $G_{2} / \mathrm{SU}(3) \quad 20$

\section{Introduction}

Instantons are important objects in modern field theories [1, 2]. Yang-Mills instantons [3] are nonperturbative Bogomolny-Prasad-Sommerfield (BPS) configurations in four Euclidean dimensions solving first-order anti-self-duality equations for gauge fields which imply the full Yang-Mills equations. They play a prominent role both in mathematics and physics, and their study has yielded many results in both areas. In this article we discuss their higher dimensional generalization.

As one motivation for our study, we notice that Yang-Mills theory in more than four dimensions naturally appears in the low-energy limit of superstring theory in the presence of D-branes. Also, heterotic strings yield heterotic supergravity, which contains supersymmetric Yang-Mills theory as a subsector [4]. Furthermore, natural BPS-type equations for gauge fields in dimension $d>4$, introduced in [5], also appear in heterotic superstring compactification on spacetimes $M_{10-d} \times X^{d}$ as the condition of survival of at least one supersymmetry in the low-energy effective field theory on $M_{10-d}$. These first-order instanton equations on $X^{d}$, which generalize the four-dimensional ones, were considered e.g. in [6-17], and some of their solutions were found in [18-33].

The Yang-Mills instantons considered here can therefore be thought of as ingredients for the construction of solitons in string theory. In heterotic string theory such solitons were 
first considered in [34-36] and were interpreted as gauge 5-brane solitons, with ordinary Yang-Mills instantons living in the four dimensions transverse to the world-volume of a flat 5-brane. Then, using heterotic - type I string duality, it was shown [37-40] that an instanton on $\mathbb{R}^{4} \subset \mathbb{R}^{10}$ shrunk to zero size $\mathrm{e}^{1}$ corresponds to a D5-brane ${ }^{2}$ in type I string theory.

Not many instanton solutions are known for $d>4$. One of them is the $\operatorname{Spin}(7)$ instanton on $\mathbb{R}^{8}$ constructed by Fairlie and Nuyts and independently by Fubini and Nicolai $[18,19]$. This solution was extended to a gauge solitonic 1-brane solution of heterotic supergravity [41], the low-energy limit of the heterotic string. A similar $G_{2}$-instanton [20-22] on $\mathbb{R}^{7}$ was extended to a heterotic 2-brane soliton independently in [42] and [22]. Due to the connection between octonions and the groups $G_{2}$ and $\operatorname{Spin}(7)$, these gauge fields on $\mathbb{R}^{7}$ and $\mathbb{R}^{8}$ are called octonionic instantons. In this paper we review recent work and present some new results showing that the octonionic instantons are part of a larger family of instantons that exist not only on Euclidean spaces but on a whole class of conical, non-compact manifolds. This generalization of the octonionic instantons to other spaces embeds into supergravity as well [43], and it is an interesting question whether this is true for the whole family of instantons. We leave this to future work, however.

A starting point for our investigation is the fact that $\mathbb{R}^{7}$ and $\mathbb{R}^{8}$ are the metric cones over the round spheres $S^{6}$ and $S^{7}$, which carry a nearly Kähler structure and a nearly parallel $G_{2}$-structure, respectively. Six-dimensional nearly Kähler SU(3)-manifolds and seven-dimensional nearly parallel $G_{2}$-manifolds have weak holonomy groups $\mathrm{SU}(3)$ and $G_{2}$, respectively. They are closely related to integrable geometries, however, as their cones have reduced holonomy groups $G_{2}$ and $\operatorname{Spin}(7)$ [44]. Furthermore, nearly Kähler SU(3)structure and nearly parallel $G_{2}$-structure are special among non-integrable geometries in that their natural instanton equations imply the usual Yang-Mills equations without a torsion term $[43,45]$, like it is the case for integrable $G$-structures.

Similar to the result that the cone has a reduced holonomy group is the observation made in $[46,47]$ that the so-called sine-cone over a nearly Kähler manifold has a nearly parallel $G_{2}$-structure. Like the cone, the sine-cone over a non-spherical manifold is singular and non-complete as a Riemannian manifold. For a round sphere, on the other hand, the cone gives Euclidean space and the sine-cone is again a sphere. Another important property is the fact that the cone over a sine-cone is the same as the cylinder over a cone [48], as illustrated for nearly Kähler manifolds in figure 1.

Given a nearly Kähler manifold $\mathcal{M}$ there are thus at least two different interesting $G_{2}$-instanton equations in one dimension higher. First, the equation on the cone with its integrable $G_{2}$-structure, and second the $G_{2}$-instanton equation on the nearly parallel sine-cone. Additionally there are two $G_{2}$-structures on the cylinder over the nearly Kähler space, but one of those is conformally equivalent to the integrable structure on the cone, and therefore does not give rise to a new instanton equation.

Of the three inequivalent $G_{2}$-instanton equations the two equations on a cylinder have been studied in a series of papers [30-33], and some explicit solutions have been found.

\footnotetext{
${ }^{1}$ Such singular instantons produce conical singularities in the instanton moduli space.

${ }^{2}$ More precisely, such gauge string solitons correspond to a bound system of D1- and D5-branes [39, 40].
} 
Here we shall present a unified approach to all these equations, by stepping up one more dimension to the cone over the sine-cone, or equivalently the cylinder over the cone, on the nearly Kähler manifold. This eight-manifold has reduced holonomy group Spin(7) and contains all of the above-mentioned seven-manifolds as submanifolds. Furthermore, the Spin(7)-instanton equation can be reduced to the different $G_{2}$-instanton equations, and therefore all the solutions found for particular $G_{2}$-structures can be considered as solutions to one single Spin(7)-instanton equation. In [30-33] a particular SU(3)-invariant ansatz for the gauge fields has been employed, which depends on one complex function of one complex variable when considered in eight dimensions. ${ }^{3}$ The Spin(7)-instanton equation turns into a first-order non-linear differential equation, with an $S_{3}$-symmetry that reflects the so-called 3-symmetry of nearly Kähler coset spaces [49].

Here we present the known solutions and also a new one. In addition, we show that our solutions include the octonionic instanton on $\mathbb{R}^{7}[20-22]$. The gauge group for these examples can either be taken to be $G_{2}$, or it can be identified with the group $G$ for a nearly Kähler coset space $G / H^{4}$ A generalized ansatz with gauge group $\operatorname{Spin}(7)$ is discussed as well, covering the octonionic instanton on $\mathbb{R}^{8}[18,19]$.

A review of the relevant geometric structures can be found in section 2, and the instanton equations are presented in section 3. Section 4 contains an alternative approach to the instanton equations in terms of a Chern-Simons action, as well as the relevant second-order equations. Our ansatz for the gauge field is explained in section 5, and in 5.2 we collect the known solutions to the $\operatorname{Spin}(7)$-instanton equation. An appendix compares the octonionic instanton on $\mathbb{R}^{7}$ to the one on the cone over $G_{2} / \mathrm{SU}(3)$.

\section{Cones, sine-cones and cylinders over nearly Kähler manifolds}

We review the geometry of six-, seven- and eight-dimensional manifolds with structure group $\mathrm{SU}(3), G_{2}$ and $\operatorname{Spin}(7)$, respectively, discuss metric cones and sine-cones over these manifolds and the structure they inherit from the base manifold.

For any Riemannian manifold $(\mathcal{M}, g)$ we define

(a) $C(\mathcal{M})=\left(\mathbb{R}^{+} \times \mathcal{M}, \bar{g}\right)$ with $\bar{g}=d r^{2}+r^{2} g$ as the Riemannian or metric cone over $\mathcal{M}$,

(b) $C_{s}(\mathcal{M})=((0, \pi) \times \mathcal{M}, \bar{g})$ with $\bar{g}=\mathrm{d} \theta^{2}+\sin ^{2}(\theta) g$ as the sine-cone over $\mathcal{M}$ and

(c) $\operatorname{Cyl}(\mathcal{M})=(\mathbb{R} \times \mathcal{M}, \bar{g})$ with $\bar{g}=d x^{2}+g$ as the cylinder over $\mathcal{M}$.

In the limits $\theta \rightarrow 0, \pi$ the sine-cone looks like the metric cone.

We can, of course, take one of these manifolds again as a base manifold for another metric cone, sine-cone or cylinder. However, it is easy to show that for two of these constructions we obtain the same manifold: the cone over a sine-cone is the same as the

\footnotetext{
${ }^{3}$ Note that more general anzätze were considered as well [32, 33].

${ }^{4}$ There is some risk of confusion here due to the different groups appearing. The manifolds under consideration come equipped with a reduced weak holonomy group, which is either $\mathrm{SU}(3), G_{2}$, or $\operatorname{Spin}(7)$, and the six-dimensional base manifold will be chosen as a homogeneous space $G / H$. Additionally, the gauge group of the gauge bundle plays a role.
} 


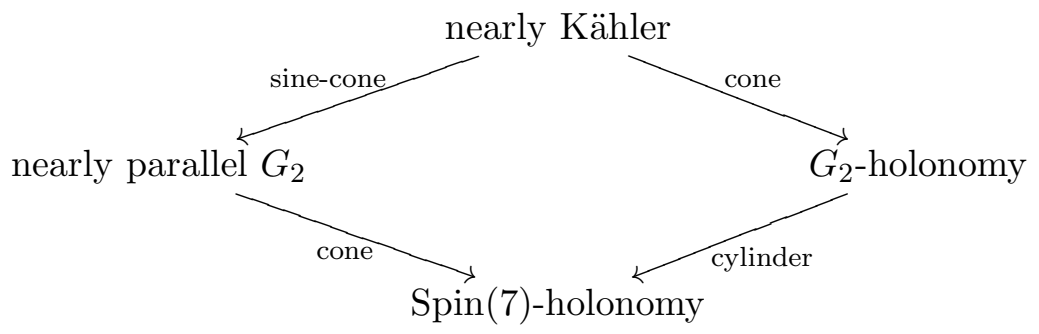

Figure 1. Cones over nearly Kähler manifolds.

cylinder over a cone, i.e. $C\left(C_{s}(\mathcal{M})\right)=C y l(C(\mathcal{M}))=\left(\mathbb{R} \times \mathbb{R}^{+} \times \mathcal{M}\right)$. The metric $\bar{g}$ of $C\left(C_{s}(\mathcal{M})\right)$ can be rewritten in terms of coordinates $(x, y)$ on $C y l(C(\mathcal{M}))$ as

$$
\begin{aligned}
\bar{g} & =\mathrm{d} r^{2}+r^{2}\left(\mathrm{~d} \theta^{2}+\sin ^{2}(\theta) g\right) \\
& =\mathrm{d} x^{2}+\mathrm{d} y^{2}+y^{2} g,
\end{aligned}
$$

where

$$
(x, y)=(r \cos (\theta), r \sin (\theta)) .
$$

Here we are interested in cone structures constructed over nearly Kähler six-manifolds. If we normalize the nearly Kähler manifold such that its Einstein constant is 5, i.e. Ric $=5 \mathrm{~g}$, then its metric cone has $G_{2}$-holonomy and its sine-cone admits a nearly parallel $G_{2}$-structure [48]. For the definition of these geometries see the following subsections. If we did not fix the normalization of the base manifold we would have to define the cone metric as $d r^{2}+\left(r / r_{0}\right)^{2} g$ and the sine-cone metric as $d \theta^{2}+\sin ^{2}\left(\theta / \theta_{0}\right) g$ for appropriately chosen constants $r_{0}, \theta_{0}$, depending only on the Einstein constant or scalar curvature of the base, to obtain $G_{2}$-holonomy and a nearly parallel $G_{2}$-structure, respectively.

From both $G_{2}$-manifolds, we can construct a $\operatorname{Spin}(7)$-holonomy manifold as summarized in figure 1 . The cone over a nearly parallel $G_{2}$-manifold and the cylinder over a $G_{2}$-holonomy manifold have $\operatorname{Spin}(7)$-holonomy. For this to be true we need to normalize the nearly parallel $G_{2}$-space as Ric $=6 g$, which comes out right automatically if it is constructed as a sine-cone over a nearly Kähler manifold normalized as above. In general the normalization condition Ric $=(\operatorname{dim} \mathcal{M}-1) g$ on an Einstein space $\mathcal{M}$ implies that its metric cone is Ricci-flat, whereas its sine-cone is Einstein again. In the special case that we start with the nearly Kähler manifold $S^{6}$, the resulting spaces are depicted in figure 2 .

\section{$2.1 \quad$ Nearly Kähler cosets $G / H$}

Manifolds of dimension six with SU(3)-structure admit a set of canonical objects fixed by the group $\mathrm{SU}(3)$, consisting of an almost complex structure $J$, a Riemannian metric $g$, a real two-form $\omega$ and a complex three-form $\Omega$. With respect to $J$, the forms $\omega$ and $\Omega$ are of type $(1,1)$ and $(3,0)$, respectively, and there is a compatibility condition, $g(J \cdot, \cdot)=\omega(\cdot, \cdot)$. 


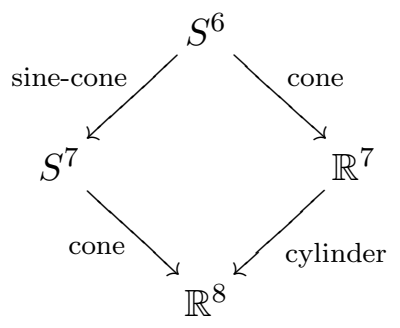

Figure 2. Cones over $S^{6}$.

With respect to the volume form $V_{g}$ of $g, \omega$ and $\Omega$ are normalized so that

$$
\omega \wedge \omega \wedge \omega=6 V_{g} \quad \text { and } \quad \Omega \wedge \bar{\Omega}=-8 \mathrm{i} V_{g} .
$$

A nearly Kähler six-manifold is an SU(3)-structure manifold such that

$$
\mathrm{d} \omega=3 \lambda \operatorname{Im} \Omega \quad \text { and } \quad \mathrm{d} \Omega=2 \lambda \omega \wedge \omega
$$

for some real non-zero constant $\lambda$, proportional to the square of the scalar curvature (if $\lambda$ was zero, the manifold would be Calabi-Yau). Our normalization Ric $=5 g$ implies that $\lambda=1$.

There are only four known examples of compact nearly Kähler six-manifolds, and all of them are coset spaces:

$$
\begin{array}{ll}
\mathrm{SU}(3) / \mathrm{U}(1) \times \mathrm{U}(1), & \mathrm{Sp}(2) /(\mathrm{Sp}(1) \times \mathrm{U}(1))_{\text {non-max }}, \\
G_{2} / \mathrm{SU}(3)=S^{6}, & \mathrm{SU}(2)^{3} / \mathrm{SU}(2)_{\operatorname{diag}}=S^{3} \times S^{3} .
\end{array}
$$

These coset spaces $G / H$ were named 3 -symmetric by Wolf and Gray, because the subgroup $H$ is the fixed point set of an automorphism $s$ of $G$ satisfying $s^{3}=\operatorname{Id}$ [49-51]. The cosets under consideration are all naturally-reductive, which means that there is a decomposition $\mathfrak{g}=\mathfrak{h} \oplus \mathfrak{m}$, orthogonal with respect to the Cartan-Killing form, where $\mathfrak{g}$ and $\mathfrak{h}$ are the Lie algebras of $G$ and $H$, respectively, and $\mathfrak{m}$ is an $\mathfrak{h}$-module: $[\mathfrak{h}, \mathfrak{m}] \subset \mathfrak{m}$. The tangent space of $G / H$ at a given point can be identified with $\mathfrak{m}$, and in particular the almost complex structure $J$ comes from an endomorphism $J: \mathfrak{m} \rightarrow \mathfrak{m}$. Similarly the 3 -symmetry induces an automorphism $S$ of the Lie algebra $\mathfrak{g}$ which acts trivially on $\mathfrak{h}$ and is related to $J$ by

$$
\left.S\right|_{\mathfrak{m}}=-\frac{1}{2}+\frac{\sqrt{3}}{2} J=\exp \left(\frac{2 \pi}{3} J\right) .
$$

The metric on $\mathfrak{m}$ is given by one twelfth times the Cartan-Killing form of $\mathfrak{g}$,

$$
g(X, Y)=-\frac{1}{12} \operatorname{Tr}_{\mathfrak{g}}(\operatorname{ad}(X) \circ \operatorname{ad}(Y))
$$

for $X, Y \in \mathfrak{m}$, and due to $g$ being $H$-invariant it extends to a globally defined metric on $G / H$. The (1,1)-form $\omega$ is fixed by its compatibility with $g$ and $J$, and $\Omega$ is the unique suitably normalized $G$-invariant (3,0)-form. 
In calculations, it is useful to choose a basis $\left\{I_{A}\right\}$ for the Lie algebra $\mathfrak{g}$. We do so in such a way that $I_{a}$ for $a=1, \ldots, 6$ form a basis for $\mathfrak{m}$ and $I_{i}$ for $i=7, \ldots, \operatorname{dim}(G)$ yield a basis for $\mathfrak{h}$. Furthermore, we impose a suitable normalization for the structure constants $f_{A B}^{C}$ :

$$
\left[I_{A}, I_{B}\right]=f_{A B}^{C} I_{C} \quad \text { with } \quad f_{A C}^{D} f_{D B}^{C}=12 \delta_{A B} .
$$

Then $f_{A B C}:=f_{A B}^{D} \delta_{D C}$ is totally antisymmetric. The reductive property of the coset means that the structure constants $f_{a i j}$ vanish. Then the 3-symmetry implies useful identities involving $\omega$ : notably, the tensor

$$
\tilde{f}_{a b c}:=f_{a b d} \omega_{d c}
$$

is totally antisymmetric; furthermore,

$$
\omega_{a c} f_{c b i}=\omega_{b c} f_{c a i} \quad \text { and } \quad \omega_{a b} f_{a b i}=0,
$$

which tell us that the endomorphisms $I_{a} \mapsto f_{i a}^{b} I_{b}$ of $\mathfrak{m}$ are contained in the $\mathfrak{s u}(3)$-subalgebra of $\mathfrak{s o}(\mathfrak{m}) \cong \mathfrak{s o}(6)$ defined by the almost complex structure.

The metric and almost complex structure on $\mathfrak{m}$ lift to a $G$-invariant metric and almost complex structure on $G / H$. Local expressions for these can be obtained by introducing an orthonormal frame as follows. The basis elements $I_{A}$ of the Lie algebra $\mathfrak{g}$ can be represented by left-invariant vector fields $\hat{E}_{A}$ on the Lie group $G$, and the dual basis $\hat{e}^{A}$ is a set of leftinvariant one-forms. The space $G / H$ consists of left cosets $g H$, and the natural projection $g \mapsto g H$ is denoted by $\pi: G \rightarrow G / H$. Over a contractible open subset $U$ of $G / H$, one can choose a map $L: U \rightarrow G$ such that $\pi \circ L$ is the identity (in other words, $L$ is a local section of the principal bundle $G \rightarrow G / H)$. The pull-backs of $\hat{e}^{A}$ under $L$ are denoted by $e^{A}$. In particular, $e^{a}$ form an orthonormal frame for $T^{*}(G / H)$ over $U$ (where again $a=1, \ldots 6$ ), and we can write $e^{i}=e_{a}^{i} e^{a}$ with real functions $e_{a}^{i}$. The dual frame for $T(G / H)$ will be denoted by $E_{a}$. The forms $e^{A}$ obey the Maurer-Cartan equations,

$$
\begin{aligned}
\mathrm{d} e^{a} & =-f_{i b}^{a} e^{i} \wedge e^{b}-\frac{1}{2} f_{b c}^{a} e^{b} \wedge e^{c}, \\
\mathrm{~d} e^{i} & =-\frac{1}{2} f_{b c}^{i} e^{b} \wedge e^{c}-\frac{1}{2} f_{j k}^{i} e^{j} \wedge e^{k} .
\end{aligned}
$$

Since all the connections we will consider are invariant under some action of $G$, it suffices to do calculations just over the subset $U$. Local expressions for the $G$-invariant metric, almost complex structure, and nearly Kähler form on $G / H$ are then

$$
g=\delta_{a b} e^{a} e^{b}, \quad J=J_{b}^{a} E_{a} e^{b} \quad \text { and } \quad \omega=\frac{1}{2} \omega_{a b} e^{a} \wedge e^{b},
$$

where in fact $\delta_{a c} J_{b}^{c}=\omega_{a b}$. One can also obtain a local expression for the $(3,0)$-form $\Omega$. From (2.11) it follows that

$$
\mathrm{d} \omega=-\frac{1}{2} \tilde{f}_{a b c} e^{a} \wedge e^{b} \wedge e^{c} \quad \text { and } \quad * \mathrm{~d} \omega=\frac{1}{2} f_{a b c} e^{a} \wedge e^{b} \wedge e^{c} .
$$

As we have $\mathrm{d} \omega=3 \operatorname{Im} \Omega$ it must be that

$$
\operatorname{Im} \Omega=-\frac{1}{6} \tilde{f}_{a b c} e^{a} \wedge e^{b} \wedge e^{c} \quad \text { and } \quad \operatorname{Re} \Omega=-\frac{1}{6} f_{a b c} e^{a} \wedge e^{b} \wedge e^{c} .
$$




\section{$2.2 \quad G_{2}$-structures}

Consider a seven-dimensional manifold with the structure group of its tangent bundle contained in $G_{2}$. The $G_{2}$-invariant objects we have at our disposal are the Riemannian metric and a real three-form $\Psi$. Locally, it is always possible to choose an orthonormal coframe $\left\{e^{1}, \ldots, e^{7}\right\}$ such that $\Psi$ can be expressed as

$$
\Psi=\frac{1}{3 !} f_{a b c}^{\mathbb{Q}} e^{a} \wedge e^{b} \wedge e^{c} \quad \text { with } \quad a, b, c=1, \ldots, 7,
$$

with $f_{a b c}^{\mathbb{Q}}$ being the octonionic structure constants. If $\Psi$ is closed and coclosed, the manifold has holonomy contained in $G_{2}$.

Cones. For nearly parallel $G_{2}$-manifolds, by definition $\Psi$ satisfies

$$
\mathrm{d} \Psi=\gamma * \Psi \quad(\text { implying } \mathrm{d} * \Psi=0)
$$

for some constant $\gamma \in \mathbb{R}$. The normalization Ric $=6 g$ implies $\gamma= \pm 4$. The $G_{2}$-manifolds that are of interest for us are cones and sine-cones $\left(\mathcal{M}^{7}, \bar{g}\right)$ on a nearly Kähler manifold $\left(\mathcal{M}^{6}, g\right)$. First, we consider the sine-cone: We can use the two- and three-forms $\omega$ and $\Omega$ on the base manifold to define a three-form

$$
\Psi^{s c}=\sin ^{2}(\theta) \omega \wedge \mathrm{d} \theta+\sin ^{3}(\theta) \operatorname{Im}\left(\mathrm{e}^{\mathrm{i} \theta} \Omega\right)
$$

on the sine-cone over $\mathcal{M}^{6}$. It satisfies $\mathrm{d} \Psi=4 * \Psi$ and induces a nearly parallel $G_{2}$-structure on the sine-cone. On the metric cone with a radial variable $y$ a three-form can be defined as

$$
\Psi^{c}=y^{2} \omega \wedge \mathrm{d} y+y^{3} \operatorname{Im} \Omega .
$$

It is closed and coclosed, reflecting the fact that the cone on a nearly Kähler manifold has $G_{2}$-holonomy. As mentioned in section 2 the metric cone can be regarded as the limit of the sine-cone for $\theta \rightarrow 0, \pi$. Equivalently, the three-form on the cone (2.18) can be obtained by considering the corresponding form (2.17) on the sine-cone in this limit.

Cylinders. There are two interesting $G_{2}$-structures on the cylinder $\mathbb{R} \times \mathcal{M}$, with its metric $\bar{g}=d \tau^{2}+g$. They are

$$
\begin{aligned}
& \Psi^{1}=\omega \wedge d \tau+\operatorname{Im} \Omega, \\
& \Psi^{2}=\omega \wedge d \tau-\operatorname{Re} \Omega .
\end{aligned}
$$

Note that the cylinder metric is conformally equivalent to the cone metric, under the substitution $y=e^{\tau}$. Under this conformal equivalence the 3 -form $\Psi^{1}$ gets identified with the 3 -form (2.18), defining the parallel $G_{2}$-structure on the cone. Therefore we call the cylinder equipped with $\Psi^{1}$ conformally parallel. The 3 -form $\Psi^{2}$ on the other hand is coclosed but neither closed nor nearly parallel, and such general $G_{2}$-structures are called cocalibrated. Manifolds with cocalibrated $G_{2}$-structures admit a compatible connection with totally skew-symmetric torsion [52], which makes them promising candidates for supergravity backgrounds. For $\theta$ close to $\pi / 2$ the sine-cone looks like a cocalibrated cylinder. 


\subsection{Spin(7)-holonomy from $G_{2}$-structure manifolds}

Consider now an eight-dimensional manifold $\mathcal{M}^{8}$. It is called a $\operatorname{Spin}(7)$-manifold if it comes equipped with a Riemannian metric $g$ and a closed self-dual four-form $\Sigma$. As illustrated in figure 1 there are two equivalent possibilities to obtain a Spin(7)-holonomy manifold $\left(\mathcal{M}^{8}, \tilde{g}\right)$ by a cone construction on a nearly Kähler manifold $\mathcal{M}^{6}$ : the metric cone over the nearly parallel $G_{2}$-manifold $C_{s}\left(\mathcal{M}^{6}\right)$ or the cylinder over the $G_{2}$-holonomy manifold $C\left(\mathcal{M}^{6}\right)$. They give rise to two convenient sets of coordinates, $(x, y) \in \mathbb{R} \times \mathbb{R}^{+}$and $(r, \theta) \in \mathbb{R}^{+} \times(0, \pi)$, related as in (2.2). In both cases, we can use the $G_{2}$-invariant three-form $\Psi$ on the base $\left(\mathcal{M}^{7}, \bar{g}\right)$ to define a four-form

$$
\begin{aligned}
\Sigma & =\mathrm{d} x \wedge \Psi^{c}+*_{7} \Psi^{c} \\
& =r^{3} \mathrm{~d} r \wedge \Psi^{s c}+r^{4} *_{7} \Psi^{s c},
\end{aligned}
$$

where the metric on $\mathcal{M}^{8}$ is

$$
\tilde{g}=\mathrm{d} x^{2}+\bar{g}^{c}=\mathrm{d} r^{2}+r^{2} \bar{g}^{s c},
$$

with $\bar{g}^{c}$ and $\bar{g}^{s c}$ the cone and sine-cone metric, respectively. A good way to see that the two four-forms coincide is by noting that they can be written as

$$
\Sigma=\frac{1}{2} \tilde{\omega} \wedge \tilde{\omega}-\operatorname{Re} \tilde{\Omega},
$$

where

$$
\tilde{\omega}=y^{2} \omega+\mathrm{d} x \wedge \mathrm{d} y \quad \text { and } \quad \tilde{\Omega}=y^{3} \Omega \wedge(\mathrm{d} y-\mathrm{i} \mathrm{d} x)
$$

in terms of the canonical coordinates on $C y l\left(C\left(\mathcal{M}^{6}\right)\right)$, or in coordinates of $C\left(C_{s}\left(\mathcal{M}^{6}\right)\right)$ :

$$
\tilde{\omega}=r^{2} \sin ^{2}(\theta) \omega+r \mathrm{~d} r \wedge \mathrm{d} \theta \quad \text { and } \quad \tilde{\Omega}=-\mathrm{i} r^{3} \sin ^{3}(\theta) \Omega \wedge \mathrm{d}\left(\mathrm{e}^{\mathrm{i} \theta} r\right) .
$$

Although we have not found a simple way to construct the 8-dimensional manifold $\operatorname{Cyl}\left(C\left(\mathcal{M}^{6}\right)\right)$ from the cylinder $C y l\left(\mathcal{M}^{6}\right)$, the latter one is contained in it as the submanifold $y=y_{0}=$ const. The relation between the different spaces is illustrated in figure 3 .

\section{Instantons and submanifolds}

We consider the instanton equation on the geometries discussed in the previous section. Let $\mathcal{E}$ be a principal $G$-bundle over a $n$-manifold $\mathcal{M}$ and $\mathcal{A}$ a connection on $\mathcal{E}$, with curvature 2-form $\mathcal{F}:=\mathrm{d} \mathcal{A}+\mathcal{A} \wedge \mathcal{A}$. The instanton equation can be written as

$$
* \mathcal{F}=-\Xi \wedge \mathcal{F}
$$

for a $(n-4)$-form $\Xi$ on $\mathcal{M}$. More precisely,

$$
\Xi=\left\{\begin{array}{lll}
\omega & \text { for } & n=6 \\
\Psi & \text { for } & n=7 \\
\Sigma & \text { for } & n=8
\end{array}\right.
$$




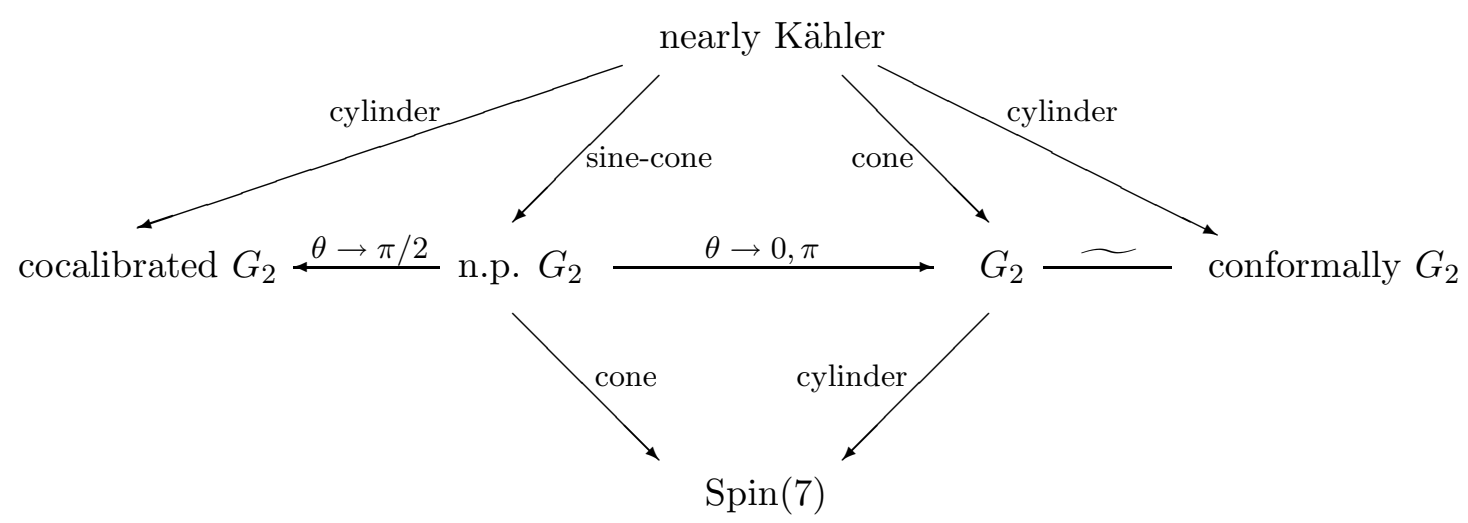

Figure 3. Metric cones, sine-cones and cylinders over nearly Kähler manifolds. The vertical position of a manifold gives its dimension, from six at the top to eight at the bottom. Here $G_{2}$ and $\operatorname{Spin}(7)$ denote holonomy groups of manifolds, and n.p. $G_{2}$ is a nearly parallel $G_{2}$-manifold. The cylinder over a nearly Kähler manifold is conformally equivalent to the cone, and therefore carries a conformally parallel $G_{2}$-structure. There is also a cocalibrated $G_{2}$-structure on the cylinder, however, which is why it occurs twice. All spaces in this diagram are submanifolds of the $\operatorname{Spin}(7)$-manifold $\mathcal{M}^{8}$, and the $\operatorname{Spin}(7)$-structure on $\mathcal{M}^{8}$ induces the cocalibrated $G_{2}$-structure on the cylinder, rather than the conformally parallel one.

where $\Psi$ and $\Sigma$ are the forms defining the $G_{2^{-}}$and $\operatorname{Spin}(7)$-structure on a seven- or eightmanifold and $\omega$ is the nearly Kähler $(1,1)$-form in six dimensions. By differentiating (3.1) it follows that instanton solutions satisfy a Yang-Mills equation with torsion

$$
D_{\mathcal{A}} * \mathcal{F}+* \mathcal{H} \wedge \mathcal{F}=0
$$

where the torsion three-form $\mathcal{H}$ is defined via $\Xi$ as

$$
* \mathcal{H}:=\mathrm{d} \Xi
$$

and

$$
D_{\mathcal{A}} * \mathcal{F}:=\mathrm{d} * \mathcal{F}+\mathcal{A} \wedge * \mathcal{F}+(-1)^{n-1} * \mathcal{F} \wedge \mathcal{A}
$$

in $n$ dimensions. On the manifolds of interest to us, however, the instanton equation also implies the Yang-Mills equation without torsion. For manifolds with $G_{2^{-}}$or $\operatorname{Spin}(7)$ holonomy this is obvious since we have $\mathrm{d} \Xi=0$ in these cases. Furthermore, it can be shown that the second term in (3.3) vanishes if $\mathcal{F}$ is an instanton on a nearly Kähler or nearly parallel $G_{2}$-manifold. In fact, the SU(3)-instanton equation on a nearly Kähler manifold can also be written as [45]

$$
\mathcal{F} \wedge \Omega=0
$$

and is equivalent to the Hermitian-Yang-Mills equation $\left.\mathcal{F}^{(2,0)}=\mathcal{F}\right\lrcorner \omega=0$. The instanton equation on a $G_{2}$-manifold on the other hand has the alternative formulation

$$
\mathcal{F}\lrcorner \Psi=0,
$$

so that in both cases instantons satisfy $\mathcal{F} \wedge \mathrm{d} \Xi=0$. 
The instanton equations (3.1) have a natural interpretation in terms of the Lie algebra $\mathfrak{k}$ of the structure group of the tangent bundle $T \mathcal{M}$. The space of two-forms on $\mathcal{M}$ at a given point is isomorphic to the Lie algebra $\mathfrak{s o}(n)$ for $\operatorname{dim} \mathcal{M}=n$. Thus, if $\mathfrak{g}$ denotes the Lie algebra of the gauge group $G, \mathcal{F}$ assumes values in $\Lambda^{2} T^{*} \mathcal{M} \otimes \mathfrak{g} \cong \mathfrak{s o}(n) \otimes \mathfrak{g}$. From this point of view, the instanton equations (3.1) are equivalent to the condition that $\mathcal{F}$ assumes values in $\mathfrak{k} \otimes \mathfrak{g} \subset \mathfrak{s o}(n) \otimes \mathfrak{g}$ with $\mathfrak{k}=\mathfrak{s u}(3), \mathfrak{g}_{2}$ or $\mathfrak{s p i n}(7)$ in dimension six, seven and eight, respectively.

In the following we will consider the Spin(7)-instanton equation on $\mathcal{M}^{8}=$ $C y l\left(C\left(\mathcal{M}^{6}\right)\right)=C\left(C_{s}\left(\mathcal{M}^{6}\right)\right)$ with $\mathcal{M}^{6}$ being a nearly Kähler coset space. Of particular interest is the reduction of this instanton equation to the submanifolds $C\left(\mathcal{M}^{6}\right), C_{s}\left(\mathcal{M}^{6}\right)$ and $C y l\left(\mathcal{M}^{6}\right)$ as well as and its relation to the $G_{2}$-instanton equations on these submanifolds. Finally, we consider the reduction of these equations to $\mathcal{M}^{6}$ and compare it to the $\mathrm{SU}(3)$-instanton equation.

Let $\mathcal{A}$ be a connection on $\mathcal{M}^{8}$ with curvature $\mathcal{F}$ satisfying the $\operatorname{Spin}(7)$-instanton equation and select an oriented submanifold $\mathcal{M}^{7}$ in $\mathcal{M}^{8}$. Later we will specify $\mathcal{M}^{7}$ to be $C\left(\mathcal{M}^{6}\right)$, $C_{s}\left(\mathcal{M}^{6}\right)$ or $C y l\left(\mathcal{M}^{6}\right)$. We denote the one-form dual to the unit normal vector of $\mathcal{M}^{7}$ by $\nu_{8}$. A generic $p$-form $\alpha$ on $\mathcal{M}^{8}$ can be decomposed as

$$
\alpha=\beta_{1}+\nu_{8} \wedge \beta_{2}
$$

with $\beta_{1} \in \Gamma\left(\Lambda^{p} T \mathcal{M}^{7}\right)$ and $\beta_{2} \in \Gamma\left(\Lambda^{p-1} T \mathcal{M}^{7}\right)$. Applying this to the $\operatorname{Spin}(7)$-instanton equation

$$
*_{8} \mathcal{F}=-\Sigma \wedge \mathcal{F}
$$

restricted to $\mathcal{M}^{7}$, it yields

$$
\begin{aligned}
*_{7} \mathcal{F} & \left.=-\Psi \wedge \mathcal{F}+\left(*_{7} \Psi\right) \wedge\left(\nu_{8}\right\lrcorner \mathcal{F}\right), \\
\left.\nu_{8}\right\lrcorner \mathcal{F} & =\mathcal{F}\lrcorner \Psi .
\end{aligned}
$$

In fact these two equations are equivalent, which can be proven by decomposing $\mathcal{F}$ according to the splitting $\mathfrak{s o}(7)=\mathfrak{g}_{2} \oplus \mathfrak{m}^{\prime}$ into its $\mathfrak{g}_{2}$ and $\mathfrak{m}^{\prime}$-components, and using the fact that the $\mathfrak{g}_{2}$-instanton operator $\mathcal{F} \mapsto *(\Psi \wedge \mathcal{F})$ has eigenvalues -1 on $\mathfrak{g}_{2}$ and 2 on $\mathfrak{m}^{\prime}$. Additionally one needs the property that $\mathcal{F} \in \mathfrak{g}_{2}$ is equivalent to $\left.\mathcal{F}\right\lrcorner \Psi=0$. Like the $G_{2}$-instanton equation, (3.10b) does not restrict the $\mathfrak{g}_{2}$-part of $\mathcal{F}$, but it allows for a non-vanishing $\mathfrak{m}^{\prime}$ component as well. In particular, for $\left.\nu_{8}\right\lrcorner \mathcal{F}=0$ the $\operatorname{Spin}(7)$-instanton equation on $\mathcal{M}^{8}$ becomes equivalent to the $G_{2}$-instanton equation on the submanifold $\mathcal{M}^{7}$.

Furthermore, we can reduce (3.10a) back to $\mathcal{M}^{6}$. We can do this equivalently from the cone $C\left(\mathcal{M}^{6}\right)$ or the sine-cone $C_{s}\left(\mathcal{M}^{6}\right)$. Analogously to the last paragraph, we denote the one-form dual to the unit normal vector to $\mathcal{M}^{6}$ by $\nu_{7}$ and split (3.10a) into three equations:

$$
\begin{aligned}
*_{6} \mathcal{F} & \left.\left.\left.\left.=-\omega \wedge \mathcal{F}-\frac{1}{2} \omega \wedge \omega\left(\nu_{7}\right\lrcorner \nu_{8}\right\lrcorner \mathcal{F}\right)-\operatorname{Im}\left(\mathrm{e}^{-\mathrm{i} \sigma} \Omega \wedge\left(\nu_{7}\right\lrcorner \mathcal{F}+\mathrm{i} \nu_{8}\right\lrcorner \mathcal{F}\right)\right), \\
\mathcal{F}\lrcorner \Omega & \left.\left.=-\mathrm{i} \mathrm{e}^{-\mathrm{i} \sigma}(\operatorname{Id}-\mathrm{i} J)\left(\nu_{7}\right\lrcorner \mathcal{F}+\mathrm{i} \nu_{8}\right\lrcorner \mathcal{F}\right), \\
3 \mathcal{F}\lrcorner \omega & \left.\left.=\nu_{7}\right\lrcorner \nu_{8}\right\lrcorner F
\end{aligned}
$$


where $\sigma$ is defined as follows. If we follow the path

$$
C y l\left(C\left(\mathcal{M}^{6}\right)\right) \rightarrow C\left(\mathcal{M}^{6}\right) \rightarrow \mathcal{M}^{6} \quad \text { we set } \quad \sigma=0,
$$

and if we reduce to $\mathcal{M}^{6}$ via

$$
C\left(C_{s}\left(\mathcal{M}^{6}\right)\right) \rightarrow C_{s}\left(\mathcal{M}^{6}\right) \rightarrow \mathcal{M}^{6} \quad \text { we set } \quad \sigma=\theta,
$$

with $\theta$ being the extra coordinate on the sine-cone. However, (3.11a) already implies (3.11b) and (3.11c). Of course, both equations could also be derived from (3.10b). We may decompose $\mathcal{F}$ as

$$
\mathcal{F}=\mathcal{F}^{2,0}+\mathcal{F}^{0,2}+\stackrel{\circ}{\mathcal{F}}^{1,1}+\mathcal{F}^{\omega} \omega
$$

where $\mathcal{F}^{2,0}$ and $\mathcal{F}^{0,2}=\overline{\mathcal{F}^{2,0}}$ are $(2,0)$ - and $(0,2)$-forms with respect to the almost complex structure $J$ while $\mathcal{F}^{1,1}$ is a $(1,1)$-form with zero $\omega$-trace. Equivalently, we can decompose (3.11a) into two equations determining the $(2,0) \oplus(0,2)$ - and $\omega$-part of $\mathcal{F}$. The $(1,1)$-part orthogonal to $\omega$ is unrestricted, as in the usual SU(3)-instanton equation. The $(2,0) \oplus(0,2)$ component of $\mathcal{F}$ is determined by (3.11b), and (3.11c) governs the $\omega$-part.

\section{Yang-Mills actions and Chern-Simons flows}

In this section we discuss an action principle that leads to the torsionful Yang-Mills equation (3.3). The action consists of the ordinary Yang-Mills action plus an additional ChernSimons term. Moreover, we clarify the relation between the pure Chern-Simons-type action on nearly Kähler or nearly parallel $G_{2}$-manifolds and the respective instanton equations.

Consider the Yang-Mills action with torsion on a manifold $\mathcal{M}$,

$$
S=S_{\mathrm{YM}}+S_{\mathrm{CS}}
$$

with

$$
S_{\mathrm{YM}}=\frac{1}{2} \int_{\mathcal{M}} \operatorname{tr}(\mathcal{F} \wedge * \mathcal{F}) \quad \text { and } \quad S_{\mathrm{CS}}=\frac{1}{2} \int_{\mathcal{M}} \operatorname{tr}(\mathcal{F} \wedge \mathcal{F} \wedge \Xi)
$$

where $\Xi$ is defined as in (3.2). The variation of $S$ yields the Yang-Mills equation with torsion

$$
D_{\mathcal{A}} * \mathcal{F}+\mathrm{d} \Xi \wedge \mathcal{F}=0
$$

which we also obtained in the previous section by differentiating the instanton equation (3.1).

On manifolds with $G_{2^{-}}$and $\operatorname{Spin}(7)$-holonomy the second term vanishes since $\Xi$ is closed in these cases. As discussed in the preceding section, for SU(3)-instantons on nearly Kähler manifolds and $G_{2}$-instantons on nearly parallel $G_{2}$-manifolds also both terms in (4.3) vanish separately. Thus, it is also enlightening to study the Chern-Simons-type action $S_{\mathrm{CS}}$ by itself, and we will consider the equation of motion obtained from $S_{\mathrm{CS}}$ on a six-dimensional nearly Kähler manifold $\mathcal{M}^{6}$ and on the sine-cone $\mathcal{M}^{7}=C\left(\mathcal{M}^{6}\right)$ over a nearly Kähler manifold. 
Chern-Simons flow on nearly Kähler manifolds. On a nearly Kähler manifold $\mathcal{M}^{6}$ we have $\Xi=\omega$, and the equation of motion resulting from $S_{\mathrm{CS}}$ is

$$
\mathrm{d} \omega \wedge \mathcal{F}=0
$$

which is exactly the $\mathrm{SU}(3)$-instanton equation. Furthermore, we can consider the gradient flow equation for the Chern-Simons-type action on $\mathcal{M}^{6}$,

$$
\frac{\mathrm{d} \mathcal{A}}{\mathrm{d} \tau}=*(\mathcal{F} \wedge \mathrm{d} \omega)
$$

where $\tau$ is a real parameter. In [31] it has been shown that (4.5) is equivalent to the $G_{2^{-}}$ instanton equation on the cylinder with the conformally parallel $G_{2}$-structure. Due to the conformal invariance of the instanton equation this is also equivalent to the $G_{2}$-instanton equation on the cone $C\left(\mathcal{M}^{6}\right)$. Moreover, it has been shown, that the Hamiltonian flow equation

$$
J \frac{\mathrm{d} \mathcal{A}}{\mathrm{d} \tau}=*(\mathcal{F} \wedge \mathrm{d} \omega)
$$

is equivalent to the $G_{2}$-instanton equation on the cocalibrated cylinder.

Chern-Simons flow on nearly parallel $G_{2}$-manifolds. On the sine-cone over $\mathcal{M}^{6}$ $(\Xi=\Psi)$ we can rewrite the equation of motion for $S_{\mathrm{CS}}$ as

$$
0=\mathrm{d} \Psi \wedge \mathcal{F}=4 * \Psi \wedge \mathcal{F},
$$

i.e. the $G_{2}$-instanton equation. Furthermore, the gradient flow equation for the ChernSimons-type action $S_{\mathrm{CS}}$ on $C_{s}\left(\mathcal{M}^{6}\right)$ is

$$
\left.\frac{\mathrm{d} \mathcal{A}}{\mathrm{d} \tau}=*(\mathcal{F} \wedge \mathrm{d} \Psi)=4 \mathcal{F}\right\lrcorner \Psi
$$

for a real parameter $\tau$. Equation (4.8) is equivalent to the $\operatorname{Spin}(7)$-instanton equation (3.10b) on $C y l\left(\mathcal{M}^{7}\right)$ and, due to the conformal invariance of the instanton equation, also to the $\operatorname{Spin}(7)$-instanton equation on the cone $C\left(\mathcal{M}^{7}\right)$.

\section{Explicit ansätze for the connection}

A number of instanton solutions on nearly Kähler, $G_{2^{-}}$and $\operatorname{Spin}(7)$-structure manifolds [30-33] are already known in the literature. Here we give a brief review of some of these solutions on cones, sine-cones and cylinders. Our starting point is the so-called canonical connection, which exists on every nearly Kähler manifold and can be written as $\hat{\mathcal{A}}=e^{i} I_{i}$ on coset spaces, using the notation of section 2.1. It has holonomy $\mathrm{SU}(3)$ and satisfies the $\mathrm{SU}(3)$-instanton equation. Instantons with gauge groups $G$ or $G_{2}$ will be found by adding further terms to $\hat{\mathcal{A}}$. We will also sketch a similar construction for gauge group $\operatorname{Spin}(7)$, starting from the canonical $G_{2}$-connection on the sine-cone. 


\subsection{Reduced instanton equations for gauge group $G$}

A generic $G$-invariant connection on $\mathcal{M}^{6}=G / H$ with gauge group equal to $G$ can be written as

$$
\mathcal{A}=e^{i} I_{i}+e^{a} \Phi_{a b} I_{b}
$$

where $\Phi$ must be $H$-invariant. We choose

$$
\Phi_{a b}=\phi_{1} \delta_{a b}+\phi_{2} \omega_{a b}
$$

with $\phi_{1,2} \in \mathbb{R}$. This is the most general possibility for $G_{2} / \mathrm{SU}(3)$; for other coset spaces further $G$-invariant gauge fields exist $[31,33]$. The curvature of this connection is given by

$$
\mathcal{F}=\frac{1}{2}\left[f_{a c i}\left(\Phi^{\top} \Phi-\mathrm{Id}\right)_{c b} I_{i}+f_{a b c}\left(-\Phi+\left(\Phi^{\top}\right)^{2}\right)_{c d} I_{d}\right] e^{a} \wedge e^{b} .
$$

For this ansatz, the $\mathrm{SU}(3)$-instanton equation is equivalent to

$$
-\phi+\bar{\phi}^{2}=0 \quad \text { where } \quad \phi:=\phi_{1}+\mathrm{i} \phi_{2} .
$$

This equation has four solutions: $\phi=0, \phi=1$ and $\phi=\exp \left( \pm \mathrm{i} \frac{2 \pi}{3}\right)$. Except for $\phi=0$ the curvature vanishes at these points. If we consider $\phi$ as a function of the additional coordinate $r, \tau$ or $\theta$ on cone, cylinder or sine-cone, respectively, then the curvature (5.3) acquires additional contributions of the form

$$
\partial_{\tau} \phi_{1} d \tau \wedge e^{a} I_{a}+\partial_{\tau} \phi_{2} d \tau \wedge e^{a} \omega_{a b} I_{b},
$$

and the $G_{2}$-instanton equation yields a differential equation for $\phi$ :

$$
\begin{aligned}
\frac{1}{2} \frac{\mathrm{d} \phi}{\mathrm{d} \tau} & =-\phi+\bar{\phi}^{2} & & \text { (conformally parallel cylinder) }, \\
\frac{\mathrm{i}}{2} \frac{\mathrm{d} \phi}{\mathrm{d} \tau} & =-\phi+\bar{\phi}^{2} & & \text { (cocalibrated cylinder) }, \\
\frac{y}{2} \frac{\mathrm{d} \phi}{\mathrm{d} y} & =-\phi+\bar{\phi}^{2} & & \text { (metric cone) }, \\
\frac{1}{2} \sin (\theta) \mathrm{e}^{-\mathrm{i} \theta} \frac{\mathrm{d} \phi}{\mathrm{d} \theta} & =-\phi+\bar{\phi}^{2} & & \text { (sine-cone) } .
\end{aligned}
$$

As explained before, the cone equation (5.6c) is equivalent to the standard instanton equation (5.6a) on the cylinder, via the substitution $y=\mathrm{e}^{\tau}$. It can be considered as a gradient flow equation, whereas the cylinder equation (5.6b) admits an interpretation as a Hamiltonian flow equation [31]. The equations (5.6) appear more naturally from an eightdimensional point of view, if we consider $\mathcal{M}^{8}=C y l\left(C\left(\mathcal{M}^{6}\right)\right)=C\left(C_{s}\left(\mathcal{M}^{6}\right)\right)$ with the metric

$$
g^{(8)}=\mathrm{d} x^{2}+\mathrm{d} y^{2}+y^{2} g^{(6)} .
$$

Choose $\mathcal{A}$ as above with $\phi$ being a complex function of $x$ and $y$. By defining a complex coordinate $z=y-\mathrm{i} x$ on $\mathbb{R}^{+} \times \mathbb{R}$, the $\operatorname{Spin}(7)$-instanton equation for this ansatz can be written as

$$
\operatorname{Re}(z) \frac{\mathrm{d} \phi}{\mathrm{d} z}=-\phi+\bar{\phi}^{2}
$$


This equation reduces to the differential equation for the cone, the sine-cone or the cocalibrated cylinder if we restrict it to a particular path in the complex half-plane spanned by $z$. One chooses

- $z=y+\mathrm{i} x_{0}$ for some constant $x_{0} \in \mathbb{R}$ to obtain the metric cone and (5.6c),

- $z=-\mathrm{i} r_{0} \mathrm{e}^{\mathrm{i} \theta}$ for some constant $r_{0}>0$ to obtain the sine-cone and (5.6d),

- $z=y_{0}-\mathrm{i} \tau$ for some constant $y_{0} \in \mathbb{R}^{+}$to obtain the cocalibrated cylinder with metric $\bar{g}=d \tau^{2}+y_{0}^{2} g^{(6)}$. The instanton equation reads

$$
\frac{\mathrm{i} y_{0}}{2} \frac{\mathrm{d} \phi}{\mathrm{d} \tau}=-\phi+\bar{\phi}^{2}
$$

which is a slight generalization of (5.6b). Contrary to the two cases above, a solution of (5.9) on the submanifold $\left\{y=y_{0}=\right.$ const $\}$ does not extend trivially to a solution on the full eight-dimensional space. This can be accomplished by choosing instead the parametrization $z=s(1-\mathrm{i} t)$. For $s$-independent $\phi$ the instanton equation becomes

$$
\frac{1}{2}(\mathrm{i}-t) \frac{\mathrm{d} \phi}{\mathrm{d} t}=-\phi+\bar{\phi}^{2},
$$

and the slices $\left\{s=s_{0}=\right.$ const $\}$ carry the cylinder metric $s_{0}^{2}\left(\mathrm{~d} t^{2}+g^{(6)}\right)$. Both (5.9) and (5.10) can be obtained from the Spin(7)-instanton equation restricted to the cylinder, as in (3.10a). In the first case one obtains exactly the cylindrical $G_{2^{-}}$ instanton equation by imposing $\left.\nu_{8}\right\lrcorner \mathcal{F}=0$, where $\nu_{8}$ is the 1 -form normal to the cylinder. This condition is not satisfied for solutions of (5.10) however, so that these do not solve the $G_{2}$-instanton equation. Substituting $t=\cot (\theta)$ in (5.10) brings us back to the sine-cone equation (5.6d).

Of course, other foliations of $\mathcal{M}^{8}$ are possible, and they lead to additional instanton equations.

Yang-Mills actions. The second-order equations obeyed by the solutions to the instanton equations admit a classical-mechanics interpretation in terms of a particle moving in the plane. It can be obtained either by inserting the ansatz (5.2) into the Yang-MillsChern-Simons action (4.1) or by differentiating the first-order equations. Up to a common prefactor of $-12 \operatorname{Vol}\left(\mathcal{M}^{6}\right)$, the action for $\phi$ becomes

$$
S_{1}^{\mathrm{cyl}}=\int_{\mathbb{R}}\left\{\frac{1}{2} \phi^{\prime} \overline{\phi^{\prime}}+V_{3}(\phi, \bar{\phi})\right\} \mathrm{d} \tau
$$

on the conformally parallel cylinder and

$$
S_{2}^{\mathrm{cyl}}=\int_{\mathbb{R}}\left\{\frac{1}{2} \phi^{\prime} \overline{\phi^{\prime}}+\operatorname{Im}\left(\phi \overline{\phi^{\prime}}\right)+V_{1}(\phi, \bar{\phi})\right\} \mathrm{d} \tau
$$

on the cocalibrated cylinder. On the metric cone and the sine-cone over $\mathcal{M}^{6}$ the action reads

$$
S^{c}=\int_{0}^{\infty}\left\{\frac{1}{2} y^{4} \phi^{\prime} \overline{\phi^{\prime}}+y^{2} V_{0}(\phi, \bar{\phi})\right\} \mathrm{d} y
$$




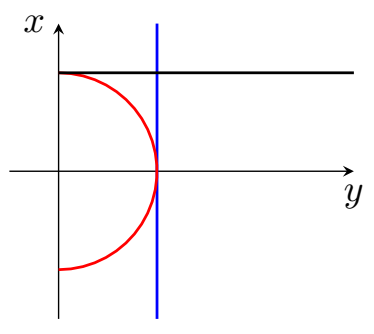

Figure 4. The complex $z$-plane, $z=y-\mathrm{i} x . \mathcal{M}^{8}$ is a twisted product of $\mathcal{M}^{6}$ with the right halfplane $\{y>0\}$. Embedded into $\mathcal{M}^{8}$ are the sine-cone (red half-circle), cylinder (vertical blue line), and cone (horizontal black line). $\mathcal{M}^{8}$ is foliated either by cylinders or cones, corresponding to the foliation of the half-plane by translations of the black and blue lines. A foliation by sine-cones is obtained through variation of the radius of the red half-circle. Upon a good parametrization of the three submanifolds the $G_{2}$-instanton equation on one of them becomes invariant under these shifts, so that a solution on a submanifold trivially extends to a $\operatorname{Spin}(7)$-instanton on all of $\mathcal{M}^{8}$.

and

$$
S^{s c}=\int_{0}^{\pi}\left\{\frac{1}{2} \sin (\theta)^{4} \phi^{\prime} \overline{\phi^{\prime}}-2 \sin (\theta)^{3} \operatorname{Re}\left[\left(-\phi+\bar{\phi}^{2}\right) \mathrm{e}^{\left.\mathrm{i} \theta \overline{\phi^{\prime}}\right]}+\sin (\theta)^{2} V_{3}(\phi, \bar{\phi})\right\} \mathrm{d} \theta,\right.
$$

respectively. Here, the potential $V_{\kappa}(\phi)$ is given by

$$
V_{\kappa}(\phi, \bar{\phi})=(\kappa-1) \phi \bar{\phi}-\left(\frac{\kappa}{3}+1\right)\left(\phi^{3}+\bar{\phi}^{3}\right)+2(\phi \bar{\phi})^{2} \quad \text { for } \quad \kappa=0,1 \text { or } 3
$$

as appropriate, and it is plotted in figure 5 below. We obtain the reduced Yang-Mills equations

$$
\begin{array}{rlrl}
\frac{\mathrm{d}^{2} \phi}{\mathrm{d} \tau^{2}} & =4 \phi-12 \bar{\phi}^{2}+8 \phi^{2} \bar{\phi}, & \text { (conf. parallel cylinder) } \\
\frac{\mathrm{d}^{2} \phi}{\mathrm{d} \tau^{2}} & =2 \mathrm{i} \frac{\mathrm{d} \phi}{\mathrm{d} \tau}-8 \bar{\phi}^{2}+8 \phi^{2} \bar{\phi}, & \text { (cocalibrated cylinder) } \\
y^{2} \frac{\mathrm{d}^{2} \phi}{\mathrm{d} y^{2}} & =-4 y \frac{\mathrm{d} \phi}{\mathrm{d} y}-2 \phi-6 \bar{\phi}^{2}+8 \phi^{2} \bar{\phi}, & \text { (metric cone) } \\
\sin (\theta)^{2} \frac{\mathrm{d}^{2} \phi}{\mathrm{d} \theta^{2}} & =-4 \sin (\theta) \mathrm{e}^{\mathrm{i} \theta}\left(\frac{\mathrm{d} \phi}{\mathrm{d} \theta}+8 \mathrm{i}\left(-\phi+\bar{\phi}^{2}\right)\right)-2 \phi-6 \bar{\phi}^{2}+8 \phi^{2} \bar{\phi} . \quad \text { (sine-cone) }
\end{array}
$$

The actions (5.11)-(5.14) and equations of motion (5.16)-(5.19) can be interpreted as describing the motion of a particle in the plane under the influence of a potential $-V_{\kappa}$ with one of the following effects:

- The equation of motion on the cocalibrated cylinder contains a term proportional to $\mathrm{i} \overline{\phi^{\prime}}$, which mimics the Lorentz force exerted on the particle by a magnetic field perpendicular to the $\phi$-plane.

- On the metric cone a friction term $-\phi^{\prime}$ appears in the equation.

- On the sine-cone both effects appear. The friction coefficient even becomes negative for $\phi>\pi / 2$, giving rise to a velocity dependent accelerating force on the particle. Moreover, the particle mass is time-dependent. 

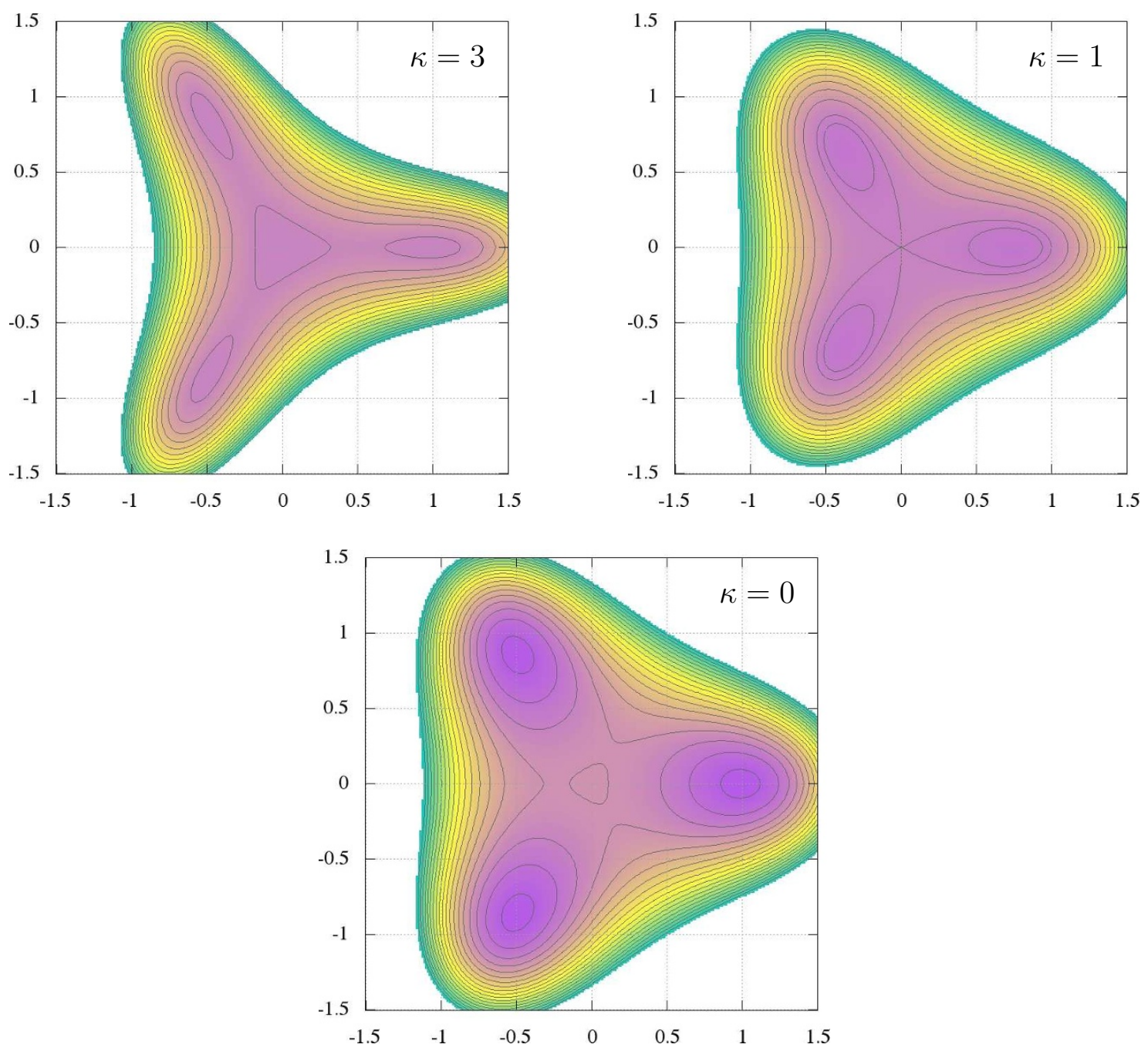

Figure 5. Contour plots of the potential $-V_{\kappa}(\phi)$ for $\kappa=3$ (top left), $\kappa=1$ (top right) and $\kappa=0$ (bottom). In all three cases, $-V_{\kappa}(\phi)$ has local maxima at $\phi=1$ and $\phi=\exp ( \pm 2 \pi \mathrm{i} / 3)$. Moreover, at $\phi=0$, the potential $-V_{3}(\phi)$ has an additional maximum on the same level as the other three maxima, $-V_{1}(\phi)$ has a saddle point, and $-V_{0}(\phi)$ has a local minimum. The friction term in the equation of motion on the cone (which has $\kappa=0$ ) leads to solutions going from one of the maxima to the minimum at $\phi=0$. For $\kappa=1$, i.e. the cocalibrated cylinder, our instanton solutions interpolate between the three maxima $\phi=1, \exp ( \pm 2 \pi \mathrm{i} / 3)$, and the same is true for the sine-cone, with $\kappa=3$. However, $\kappa=3$ also covers the conformally parallel cylinder, which only admits solutions between $\phi=0$ and one of the three other maxima.

\subsection{Solutions for gauge group $G$}

Here we collect the known finite-action solutions to the instanton equations (5.6). The conformally parallel cylinder, and thus the cone, was discussed in [30-33]:

$$
\phi(\tau)=\frac{c}{2}\left(1-\tanh \left(\tau-\tau_{0}\right)\right) \quad \text { with } \quad c=1 \quad \text { or } \quad c=\exp \left( \pm \mathrm{i} \frac{2 \pi}{3}\right)
$$




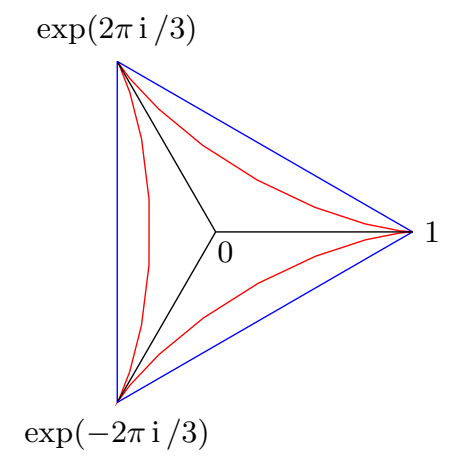

Figure 6. Instantons in the complex $\phi$ plane. The nodes correspond to the four SU(3)-instantons on the nearly Kähler manifold $\mathcal{M}^{6}$, whereas the edges are interpolating $\operatorname{Spin}(7)$-instantons on $\mathcal{M}^{8}=\operatorname{Cyl}\left(C\left(\mathcal{M}^{6}\right)\right)$ or submanifolds thereof. The blue edges can be realized as $G_{2}$-instantons on the cocalibrated cylinder $C y l\left(\mathcal{M}^{6}\right)$, the red ones solve the nearly parallel $G_{2}$-instanton equation on the sine-cone $C_{s}\left(\mathcal{M}^{6}\right)$, and the black edges are solutions on the cone $C\left(\mathcal{M}^{6}\right)$. The 3-symmetry of the nearly Kähler manifold is reflected in the permutation symmetry of the diagram.

The solution (5.20) interpolates between the stationary SU(3)-instantons $\phi \rightarrow c$ for $\tau \rightarrow$ $-\infty$ and $\phi \rightarrow 0$ for $\tau \rightarrow \infty$, and it is represented by the black edges in figure 6 . Solutions to $(5.6 \mathrm{~b})$ on the cocalibrated cylinder have been found in [31] and are given by

$$
\phi(\tau)=-\frac{c}{2}\left(1+\mathrm{i} \sqrt{3} \tanh \left[\sqrt{3}\left(\tau-\tau_{0}\right)\right]\right),
$$

with $c$ as above being equal to one of the three non-trivial fixed points. These solutions interpolate between the fixed points $c \cdot \exp (-2 \pi \mathrm{i} / 3)$ and $c \cdot \exp (2 \pi \mathrm{i} / 3)$, as is illustrated by the blue edges in figure 6 . We also found solutions for the sine-cone equation (5.6d), namely

$$
\phi(\theta)=c\left(\cos (\theta)-\frac{\mathrm{i}}{3} \sin (\theta)\right) \mathrm{e}^{\mathrm{i} \theta / 3} .
$$

These are drawn in red in figure 6 and interpolate between $c$ for $\theta \rightarrow 0$ and $c \cdot \exp (-2 \pi \mathrm{i} / 3)$ for $\theta \rightarrow \pi$. Moreover, they give rise to solutions of the $\operatorname{Spin}(7)$-instanton equation (5.10) restricted to the cylinder, upon substituting $\theta=\operatorname{arccot}(t)$. Explicitly, we get

$$
\phi(t)=c \frac{\left(t-\frac{\mathrm{i}}{3}\right)(t+\mathrm{i})^{1 / 3}}{\left(t^{2}+1\right)^{2 / 3}} .
$$

\subsection{Gauge groups $G_{2}$ and $\operatorname{Spin}(7)$}

The ansatz for the gauge field chosen above relies on the fact that on a reductive homogeneous space $\mathcal{M}=G / H$ with Lie-algebra splitting $\mathfrak{g}=\mathfrak{h} \oplus \mathfrak{m}$ we can identify the tangent space $T_{x} \mathcal{M}$ with the $H$-module $\mathfrak{m}$. Stated more globally, we have an isomorphism of vector bundles $T \mathcal{M}=G \times_{H} \mathfrak{m}$, and $H$ plays the role of the structure group of $T \mathcal{M}$. On the other hand, the nearly Kähler spaces carry an SU(3)-structure with corresponding SU(3)-frame bundle $P$, and we can also identify $T \mathcal{M}=P \times_{\mathrm{SU}(3)} \mathfrak{m}$. In fact, $H$ is a subgroup of $\mathrm{SU}(3)$ for 
nearly Kähler manifolds, and $\mathfrak{m}$ carries a SU(3)-module structure which upon restriction to $H$ gives back the action of $H$ on $\mathfrak{m}$. Hence, we meet the two reductive decompositions

$$
\mathfrak{g}=\mathfrak{h} \oplus \mathfrak{m} \quad \text { and } \quad \mathfrak{g}_{2}=\mathfrak{s u}(3) \oplus \mathfrak{m},
$$

and thus we can view $\mathfrak{m}$ either as a subspace of $\mathfrak{g}$ or of $\mathfrak{g}_{2}$. In the previous subsection we constructed several gauge fields on $C y l\left(C\left(\mathcal{M}^{6}\right)\right)$, the cylinder over the cone over a nearly Kähler six-manifold, which contain terms of the form $e^{a} \Phi_{a b} I_{b}$. Locally, the $I_{a}$ are elements of $\mathfrak{m}$, considered as a subspace of $\mathfrak{g}$ so far. According to the above discussion we can also view them as elements of $\mathfrak{g}_{2}$ and thus obtain connections with gauge group $G_{2}$. Since the instanton equations remain unchanged, this is merely a reinterpretation of the earlier results. In the case of $S^{6}=G_{2} / \mathrm{SU}(3)$ nothing changes at all.

This new interpretation has some nice features however. First of all, it is valid not only for homogeneous spaces but also, for instance, on nearly Kähler manifolds obtained as sine-cones over Sasaki-Einstein 5-manifolds [53]. Additionally, it provides a geometric interpretation for some of the instantons. Both nearly Kähler and nearly parallel $G_{2}$-manifolds possess a so-called canonical connection, which has holonomy $\mathrm{SU}(3)$ or $G_{2}$, respectively, has totally skew-symmetric torsion and satisfies the instanton equation. On manifolds with reduced holonomy group $G_{2}$ or $\operatorname{Spin}(7)$ on the other hand, the Levi-Civita connection provides one particular solution to the corresponding instanton equation.

Consider again figure 1. The four spaces with reduced structure group SU(3), $G_{2}$ or $\operatorname{Spin}(7)$ are related by certain geometric operations, and each carries a distinguished instanton. As a gauge field, the Levi-Civita connection on a Riemannian manifold can be identified with the one on its cylinder, because their connection 1-forms (or gauge fields) are the same. Thus we end up with four geometries giving rise to three different instantons. They correspond to the canonical connection on the base $\mathcal{M}$ for $\phi=0$, the Levi-Civita connection on the cone $C(\mathcal{M})$ for $\phi=1$, and additionally the canonical $G_{2}$-connection on the sine-cone $C_{s}(\mathcal{M})$, which is gauge-equivalent to the non-stationary solution (5.22). The solution (5.20) interpolating between $\phi=0$ and $\phi=1$ therefore related the pull-back of the canonical SU(3)-connection on $\mathcal{M}^{6}$ with the Levi-Civita connection on the cone $C\left(\mathcal{M}^{6}\right)$.

In the special case $\mathcal{M}^{6}=S^{6}$ the cone is simply $\mathbb{R}^{7} \backslash\{0\}$, and (5.20) reproduces the octonionic instanton [22]. This follows from the fact that our ansatz gives the most general $G_{2}$-invariant gauge field on $\mathbb{R}^{7}$, while the octonionic instanton is $G_{2}$-invariant as well. ${ }^{5} \mathrm{In}$ the appendix we verify explicitly the coincidence of the curvature tensors.

To make the identification of the gauge field for $\phi=1$ with the Levi-Civita connection on the cone more precise, we consider the following explicit realization of the $\mathfrak{m}$-generators $I_{a}$ as elements of $\mathfrak{g}_{2} \subset \mathfrak{s o}(7)$. Let indices $a, b, c$ run from 1 to 6 , and define skew-symmetric $7 \times 7$-matrices $I_{a}$ by

$$
\left(I_{a}\right)_{b 7}=\delta_{a b} \quad \text { and } \quad\left(I_{a}\right)_{b c}=\frac{1}{2}(\operatorname{Re} \Omega)_{a b c} .
$$

The cone metric can be written as $\mathrm{e}^{2 \tau}\left(d \tau^{2}+g^{6}\right)$, where $\tau$ is the logarithm of the radial coordinate, $y=\mathrm{e}^{\tau}$. In the orthonormal frame $\left\{\mathrm{e}^{\tau} e^{a}, \mathrm{e}^{\tau} d \tau\right\}$ its Levi-Civita connection

\footnotetext{
${ }^{5}$ We thank Derek Harland for pointing out this argument.
} 
assumes the form

$$
\Gamma=\hat{A}^{6}+e^{a} I_{a},
$$

where $\hat{A}^{6}=e^{i} I_{i}$ is the canonical connection of the base and the $I_{a}$ are given by the matrices (5.25) acting on the tangent space.

The canonical connection $\hat{A}$ on a nearly Kähler or nearly parallel $G_{2}$-manifold is obtained from its Levi-Civita connection by adding a suitable multiple of the canonical 3 -form $\operatorname{Re}(\Omega)$ or $\Psi$, respectively [52]. For the sine-cone over a nearly Kähler manifold this recipe leads to the following expression:

$$
\hat{A}^{7}=\hat{A}^{6}+\left[\cos (\theta) \delta_{a b}-\frac{1}{3} \sin (\theta) \omega_{a b}\right] e^{a} I_{b}-\frac{1}{3} \mathrm{~d} \theta J,
$$

where $J$ is the almost complex structure acting non-trivially only on the tangent space to $\mathcal{M}^{6}$ and the $I_{a}$ are skew-symmetric $7 \times 7$-matrices

$$
\left(I_{a}\right)_{b 7}=\delta_{a b} \quad \text { and } \quad\left(I_{a}\right)_{b c}=\frac{1}{2} \operatorname{Re}\left(\mathrm{e}^{\mathrm{i} \theta} \Omega\right)_{a b c} .
$$

Again, indices $a, b, c$ run from 1 to 6 . Upon a gauge transformation

$$
\left(\mathrm{d}+\hat{A}^{7}\right) \mapsto \mathrm{e}^{-\theta J / 3}\left(\mathrm{~d}+\hat{A}^{7}\right) \mathrm{e}^{\theta J / 3},
$$

the connection form is transferred to the form of our ansatz (5.2), with $\phi$ given by (5.22) and the $I_{a}$ by (5.25).

Now we turn to gauge group $\operatorname{Spin}(7)$. Similarly to the decomposition $\mathfrak{g}_{2}=\mathfrak{s u}(3) \oplus \mathfrak{m}$ we have a decomposition

$$
\mathfrak{s p i n}(7)=\mathfrak{g}_{2} \oplus \mathfrak{m}^{\prime},
$$

where $\mathfrak{m}^{\prime}$ is the seven-dimensional irreducible $\mathfrak{g}_{2}$-module. We can introduce a gauge field in the form

$$
A=\hat{A}^{7}+\phi \hat{e}^{a} \hat{I}_{a},
$$

where $\hat{I}_{a}$ are the generators of $\mathfrak{m}^{\prime}$ and $\hat{e}^{a}$ the dual 1-forms. As before, $\hat{A}^{7}$ denotes the canonical $G_{2}$-connection on the sine-cone $C_{s}\left(\mathcal{M}^{6}\right)$; it can be written as $\hat{A}^{7}=\hat{e}^{i} \hat{I}_{i}$ for generators $\hat{I}_{i}$ of $\mathfrak{g}_{2}$. The $\operatorname{Spin}(7)$-instanton equation for $\phi$ turns out to coincide with (5.6a) if we substitute $r=\mathrm{e}^{\tau}$, and therefore a solution is given by

$$
\phi(\tau)=\frac{1}{2}\left(1-\tanh \left(\tau-\tau_{0}\right)\right) .
$$

The gauge field interpolates between the canonical nearly parallel $G_{2}$-connection and the Levi-Civita connection on the cone. For $\mathcal{M}^{6}=S^{6}$ we have $\mathcal{M}^{8}=\mathbb{R}^{8} \backslash \mathbb{R}$, and (5.32) gives rise to the octonionic instanton $[18,19]$.

\section{Conclusions and outlook}

For a given nearly Kähler manifold $\mathcal{M}^{6}$, there are several interesting related geometries in dimension seven and eight with $G_{2^{-}}$and $\operatorname{Spin}(7)$-structures, respectively. They give rise to different instanton equations, and we have presented finite-action solutions to 
all of them, mostly by collecting earlier results. A unified approach to these equations has been developed by embedding all these seven-manifolds into the Spin(7)-manifold $\mathcal{M}^{8}=C\left(C_{s}\left(\mathcal{M}^{6}\right)=C y l\left(C\left(\mathcal{M}^{6}\right)\right)\right.$. The eight-dimensional $\operatorname{Spin}(7)$-instanton equation (5.8) induces the respective $G_{2}$-instanton equations upon restriction to the cone or the sine-cone over $\mathcal{M}^{6}$, but assumes a somewhat more general form on the cylinder, which we have been able to solve as well.

Ultimately we would like to understand the moduli spaces of $G$-invariant instantons on $\mathcal{M}^{8}$ built over an arbitrary homogeneous space $\mathcal{M}^{6}=G / H$, and for any fixed gauge group. The results presented here can be understood as a first step in this direction, by restricting attention to instantons that are invariant under translations in one direction. Several non-trivial solutions of this type exist, and the full moduli space is certainly much larger.

Among the instantons presented here are the octonionic instantons on $\mathbb{R}^{7}$ and $\mathbb{R}^{8}$. It is well-known that they can be lifted to solutions of heterotic supergravity, and in fact this is true for the $G_{2^{-}}$and $\operatorname{Spin}(7)$-instantons (5.20) and (5.32) for an arbitrary nearly Kähler base manifold [43]. An interesting question arises whether this is also possible for the instantons on the coclibrated cylinder and the sine-cone.

Most investigations of moduli spaces of heterotic string vacua so far have focussed on solutions without fluxes, based on integrable geometries like Calabi-Yaus, $G_{2^{-}}$or $\operatorname{Spin}(7)$ manifolds. The gauge field is normally required to coincide with the Levi-Civita connection in these cases. Our investigation shows that the gauge sector of heterotic string theory on certain conical Spin(7)-manifolds admits several deformations away from the Levi-Civita connection. If these embed into string theory, the moduli space of integrable backgrounds captures only a small fraction of the full vacuum structure of heterotic string theory.

A construction similar to the one presented here for six-dimensional nearly Kähler manifolds should be possible for a five-dimensional Sasaki-Einstein manifold. In this case the cone is a Calabi-Yau 3-fold, the sine-cone is a nearly Kähler manifold, and the cone over the sine-cone has $G_{2}$-holonomy group $[48,53]$. Then one could go ahead and consider also the sine-cone over the sine-cone, which is a nearly parallel $G_{2}$-manifold, and so on. It turns out, however, that the instanton equation on the cone over a Sasaki-Einstein manifold assumes a more complicated form than is the case for nearly Kähler manifolds, which could be an obstacle to obtaining explicit solutions [43].

\section{Acknowledgments}

We thank Derek Harland for helpful comments. This work was supported in part by the cluster of excellence EXC 201 "Quantum Engineering and Space-Time Research", by the Deutsche Forschungsgemeinschaft (DFG), by the Russian Foundation for Basic Research (grants RFBR 09-02-91347 and 10-01-00178) and by the Heisenberg-Landau program.

\section{A Comparing the instanton on $\mathbb{R}^{7}$ and on the cone over $G_{2} / \mathrm{SU}(3)$}

Since $G_{2} / \mathrm{SU}(3)$ with its nearly Kähler metric is the round six-sphere, the cone $C\left(G_{2} / \mathrm{SU}(3)\right)$ gives $\mathbb{R}^{7}$. In this appendix we compare explicitly the octonionic instanton on $\mathbb{R}^{7}$, constructed in [22], with the instanton (5.20) on the cone and show that they coincide. 
$G_{2}$-structure on $\mathbb{R}^{7}$ and $\mathrm{SU}(3)$-structure on $\boldsymbol{S}^{6}$. On $\mathbb{R}^{7}$ we have a canonical integrable $G_{2}$-structure

$$
\Psi=\frac{1}{6} f_{\hat{a} \hat{b} \hat{c}}^{\mathbb{Q}} \mathrm{d} x^{\hat{a}} \wedge \mathrm{d} x^{\hat{b}} \wedge \mathrm{d} x^{\hat{c}},
$$

with $x^{\hat{a}}(\hat{a}=1, \ldots, 7)$ being Euclidean coordinates on $\mathbb{R}^{7}$. This $G_{2}$-structure induces an $\mathrm{SU}(3)$-structure on $S^{6}$ defined by the forms

$$
\left.\left.\omega=\imath^{*}(\mathrm{~d} r\lrcorner \Psi\right) \quad \text { and } \quad \Omega=\imath^{*}(\mathrm{~d} r\lrcorner * \Psi\right)+\mathrm{i} \imath^{*} \Psi,
$$

where $r^{2}=x^{\hat{a}} x^{\hat{a}}$ and $\imath: S^{6} \rightarrow \mathbb{R}^{7}$ is the inclusion map of the six-sphere. The two- and three-forms $\omega$ and $\Omega$ define a nearly Kähler structure on $S^{6}$ [54]. Since up to isometry there is only one nearly Kähler structure on $S^{6}$ [55], this has to be the same structure we considered on $G_{2} / \mathrm{SU}(3)$.

Seven-dimensional representation of $\boldsymbol{G}_{\mathbf{2}}$. In [22] generators of $G_{2}$ in the sevendimensional representation were constructed via the spinor representation of $\mathrm{SO}(7)$. The generators of $\mathrm{SO}(7)$ in the spinor representation are given in terms of the gamma matrices in seven dimensions by

$$
\Gamma_{\hat{a} \hat{b}}:=\Gamma_{[\hat{a}} \Gamma_{\hat{b}]} \quad \text { with } \quad \hat{a}, \hat{b}=1, \ldots, 7 .
$$

The subgroup $G_{2} \subset \mathrm{SO}(7)$ is generated by the subset of generators $\left\{G_{\hat{a} \hat{b}}\right\} \subset\left\{\Gamma_{\hat{a} \hat{b}}\right\}$ satisfying the constraints

$$
f_{\hat{a} \hat{b} \hat{c}}^{\mathbb{Q}} G_{\hat{b} \hat{c}}=0 .
$$

Their commutation relation inherited from $\mathrm{SO}(7)$ is

$$
\left[G_{\hat{a} \hat{b}}, G_{\hat{c} \hat{d}}\right]=2 \delta_{\hat{c}[\hat{b}} G_{\hat{a}] \hat{d}}-2 \delta_{\hat{d}[\hat{b}} G_{\hat{a}] \hat{c}}+\frac{1}{2}\left((* \Psi)_{\hat{c} \hat{d} \hat{e}[\hat{a}} G_{\hat{b}] \hat{e}}-(* \Psi)_{\hat{a} \hat{b} \hat{e}[\hat{c}} G_{\hat{d}] \hat{e}}\right),
$$

and they are normalized such that

$$
\operatorname{tr}\left(G_{\hat{a} \hat{b}} G^{\hat{c} \hat{d}}\right)=-6\left[\delta_{\hat{a}}^{\hat{c}} \delta_{\hat{b}}^{\hat{d}}+\frac{1}{4}(* \Psi)_{\hat{a} \hat{b}}^{\hat{c} \hat{d}}\right] .
$$

Instanton solution on $\mathbb{R}^{7}$. The instanton solution constructed in [22] reads

$$
\begin{aligned}
& \mathcal{A}=G_{\hat{a} \hat{b}} f_{\hat{b}}(x) \mathrm{d} x^{\hat{a}} \longrightarrow \\
& \mathcal{F}=\left[2 f_{\hat{c}[\hat{a}} G_{\hat{b}] \hat{c}}-2 f_{\hat{c}} G_{\hat{c}[\hat{a}} f_{\hat{b}]}-G_{\hat{a} \hat{b}} f_{\hat{c}} f_{\hat{c}}-\frac{1}{2}(* \Psi)_{\hat{a} \hat{b} \hat{c} \hat{d}} f_{\hat{e}} G_{\hat{e} \hat{c}} f_{\hat{d}}\right] \mathrm{d} x^{\hat{a}} \wedge \mathrm{d} x^{\hat{b}},
\end{aligned}
$$

with

$$
f_{\hat{a}}=\partial_{\hat{a}} f \quad \text { and } \quad f_{\hat{a} \hat{b}}=\partial_{\hat{a}} \partial_{\hat{b}} f \quad \text { for } \quad f(y)=-\frac{1}{2} \log \left(\rho^{2}+r^{2}\right),
$$

where $\rho$ is an arbitrary scale parameter. In order to compare this solution with the solutions on the cone over $G_{2} / \mathrm{SU}(3)$, it is necessary to chose an orthonormal coframe $\left\{e^{\hat{a}}\right\}$ such that $e^{7}=\mathrm{d} r$ and the one-forms $e^{a}$ are the left-invariant one-forms defined in section 2.1. We 
consider $\mathrm{SU}(3)$ to be embedded in $G_{2}$ such that it is the stabilizer of $\mathrm{d} r$. This implies that we can write the matrices $G_{\hat{a} \hat{b}}$ in this frame as

$$
G_{a b}=\frac{1}{2} \sqrt{3} f_{a b c} \check{I}_{c}+\sqrt{3} f_{a b i} \check{I}_{i} \quad \text { and } \quad G_{a 7}=\sqrt{3} \delta_{a b} \check{I}_{b}
$$

where $\check{I}_{i}$ and $\check{I}_{a}$ are the generators of $\mathfrak{s u}(3)$ and $\mathfrak{s u}(3)^{\perp}$, respectively, in the sevendimensional representation. The matrices defined by (A.10) satisfy the commutation relation (A.5) and are compatible with (A.6) if the generators $\check{I}_{A}$ are normalized such that

$$
\operatorname{tr}\left(\check{I}_{A} \check{I}_{B}\right)=-\delta_{A B}
$$

The curvature (A.8) written in this frame reads

$$
F_{a 7}=-\frac{2 \sqrt{3} \rho^{2}}{\left(\rho^{2}+r^{2}\right)^{2}} \check{I}_{a} \quad \text { and } \quad F_{a b}=\frac{\sqrt{3}}{\left(\rho^{2}+r^{2}\right)^{2}}\left[-\left(2 \rho^{2}+r^{2}\right) f_{a b i} \check{I}_{i}-\rho^{2} f_{a b c} \check{I}_{c}\right]
$$

and it coincides with the one of the connection (5.20) for $c=1$. This completes the proof.

\section{References}

[1] R. Rajaraman, Solitons and Instantons: An introduction to solitons and instantons in quantum field theory, North-Holland, Amsterdam The Netherlands (1982) [SPIRES].

[2] N.S. Manton and P. Sutcliffe, Topological solitons, Cambridge University Press, Cambridge U.K. (2004) [SPIRES].

[3] A.A. Belavin, A.M. Polyakov, A.S. Schwartz and Y.S. Tyupkin, Pseudoparticle solutions of the Yang-Mills equations, Phys. Lett. B 59 (1975) 85 [SPIRES].

[4] M.B. Green, J.H. Schwarz and E. Witten, Superstring theory: Volumes 1 \& 2, Cambridge University Press, Cambridge U.K. (1987).

[5] E. Corrigan, C. Devchand, D.B. Fairlie and J. Nuyts, First Order Equations for Gauge Fields in Spaces of Dimension Greater Than Four, Nucl. Phys. B 214 (1983) 452 [SPIRES].

[6] R.S. Ward, Completely Solvable Gauge Field Equations in Dimension Greater Than Four, Nucl. Phys. B 236 (1984) 381 [SPIRES].

[7] S.K. Donaldson, Anti-self-dual Yang-Mills connections on a complex algebraic surface and stable vector bundles, Proc. Lond. Math. Soc. 50 (1985) 1.

[8] S.K. Donaldson, Infinite determinants, stable bundles and curvature, Duke Math. J. 54 (1987) 231.

[9] K.K. Uhlenbeck and S.-T. Yau, On the existence of Hermitian-Yang-Mills connections on stable bundles over compact Kähler manifolds, Commun. Pure Appl. Math. 39 (1986) 257.

[10] M. Mamone Capria and S.M. Salamon, Yang-Mills fields on quaternionic spaces, Nonlinearity 1 (1988) 517.

[11] R. Reyes Carrión, A generalization of the notion of instanton, Diff. Geom. Appl. 8 (1998) 1 [SPIRES].

[12] G. Tian, Gauge theory and calibrated geometry. I, Ann. Math. 151 (2000) 193 [math/0010015]. 
[13] T. Tao and G. Tian, A singularity removal theorem for Yang-Mills fields in higher dimensions, J. Amer. Math. Soc. 17 (2004) 557 [math/0209352].

[14] S.K. Donaldson and R.P. Thomas, Gauge theory in higher dimensions, in The Geometric Universe, Oxford University Press, Oxford U.K. (1998)

http://www.ma.ic.ac.uk/ rpwt/skd.pdf.

[15] S. Donaldson and E. Segal, Gauge Theory in higher dimensions, II, arXiv:0902.3239 [SPIRES].

[16] A.D. Popov, Non-Abelian Vortices, super-Yang-Mills Theory and Spin(7)-Instantons, Lett. Math. Phys. 92 (2010) 253 [arXiv:0908.3055] [SPIRES].

[17] A.D. Popov and R.J. Szabo, Double quiver gauge theory and nearly Kähler flux compactifications, arXiv:1009.3208 [SPIRES].

[18] D.B. Fairlie and J. Nuyts, Spherically symmetric solutions of gauge theories in eight dimensions, J. Phys. A 17 (1984) 2867 [SPIRES].

[19] S. Fubini and H. Nicolai, The octonionic instanton, Phys. Lett. B 155 (1985) 369 [SPIRES].

[20] T.A. Ivanova and A.D. Popov, Self-dual Yang-Mills fields in D=7,8, octonions and Ward equations, Lett. Math. Phys. 24 (1992) 85 [SPIRES].

[21] T.A. Ivanova and A.D. Popov, (Anti)selfdual gauge fields in dimension $d \geq 4$, Theor. Math. Phys. 94 (1993) 225 [SPIRES].

[22] M. Günaydin and H. Nicolai, Seven-dimensional octonionic Yang-Mills instanton and its extension to an heterotic string soliton, Phys. Lett. B 351 (1995) 169 [SPIRES].

[23] O. Lechtenfeld, A.D. Popov and R.J. Szabo, Noncommutative instantons in higher dimensions, vortices and topological K-cycles, JHEP 12 (2003) 022 [hep-th/0310267] [SPIRES].

[24] A.D. Popov, A.G. Sergeev and M. Wolf, Seiberg-Witten monopole equations on noncommutative $R^{4}$, J. Math. Phys. 44 (2003) 4527 [hep-th/0304263] [SPIRES].

[25] O. Lechtenfeld, A.D. Popov and R.J. Szabo, SU(3)-equivariant quiver gauge theories and nonabelian vortices, JHEP 08 (2008) 093 [arXiv:0806.2791] [SPIRES].

[26] T. Rahn, Yang-Mills Equations of Motion for the Higgs Sector of SU(3)-Equivariant Quiver Gauge Theories, J. Math. Phys. 51 (2010) 072302 [arXiv:0908.4275] [SPIRES].

[27] A.S. Haupt, T.A. Ivanova, O. Lechtenfeld and A.D. Popov, Chern-Simons flows on Aloff-Wallach spaces and Spin(7)-instantons, Phys. Rev. D 83 (2011) 105028 [arXiv:1104.5231] [SPIRES].

[28] F.P. Correia, Hermitian Yang-Mills instantons on Calabi-Yau cones, JHEP 12 (2009) 004 [arXiv: 0910.1096] [SPIRES].

[29] F.P. Correia, Hermitian Yang-Mills instantons on resolutions of Calabi-Yau cones, JHEP 02 (2011) 054 [arXiv: 1009.0526] [SPIRES].

[30] T.A. Ivanova, O. Lechtenfeld, A.D. Popov and T. Rahn, Instantons and Yang-Mills Flows on Coset Spaces, Lett. Math. Phys. 89 (2009) 231 [arXiv:0904.0654] [SPIRES].

[31] D. Harland, T.A. Ivanova, O. Lechtenfeld and A.D. Popov, Yang-Mills flows on nearly Kähler manifolds and $G_{2}$-instantons, Commun. Math. Phys. 300 (2010) 185 [arXiv:0909.2730] [SPIRES]. 
[32] D. Harland and A.D. Popov, Yang-Mills fields in flux compactifications on homogeneous manifolds with $\mathrm{SU}(4)$-structure, arXiv: 1005. 2837 [SPIRES].

[33] I. Bauer, T.A. Ivanova, O. Lechtenfeld and F. Lubbe, Yang-Mills instantons and dyons on homogeneous $G_{2}$-manifolds, JHEP 10 (2010) 044 [arXiv:1006.2388] [SPIRES].

[34] A. Strominger, Heterotic solitons, Nucl. Phys. B 343 (1990) 167 [Erratum ibid B 353 (1991) 565] [SPIRES].

[35] C.G. Callan Jr., J.A. Harvey and A. Strominger, World sheet approach to heterotic instantons and solitons, Nucl. Phys. B 359 (1991) 611 [SPIRES].

[36] C.G. Callan Jr., J.A. Harvey and A. Strominger, Worldbrane actions for string solitons, Nucl. Phys. B 367 (1991) 60 [SPIRES].

[37] E. Witten, Small Instantons in String Theory, Nucl. Phys. B 460 (1996) 541 [hep-th/9511030] [SPIRES].

[38] J. Polchinski and E. Witten, Evidence for heterotic - type I string duality, Nucl. Phys. B 460 (1996) 525,[hep-th/9510169] [SPIRES].

[39] M.R. Douglas, Branes within branes, hep-th/9512077 [SPIRES].

[40] M.R. Douglas, Gauge fields and D-branes, J. Geom. Phys. 28 (1998) 255 [hep-th/9604198] [SPIRES].

[41] J.A. Harvey and A. Strominger, Octonionic superstring solitons, Phys. Rev. Lett. 66 (1991) 549 [SPIRES].

[42] T.A. Ivanova, Octonions, selfduality and strings, Phys. Lett. B 315 (1993) 277 [SPIRES].

[43] D. Harland and C. Nölle, Instantons and Killing spinors, arXiv:1109.3552 [SPIRES].

[44] C. Bär, Real Killing spinors and holonomy, Commun. Math. Phys. 154 (1993) 509.

[45] F. Xu, Geometry of SU(3) manifolds, Ph.D. Thesis, Duke University, Durham U.S.A. (2008).

[46] R. Cleyton and A. Swann, Cohomogeneity-one GQ-structures, J. Geom. Phys. 44 (2002) 202 [math/0111056].

[47] A. Bilal and S. Metzger, Compact weak $G_{2}$-manifolds with conical singularities, Nucl. Phys. B 663 (2003) 343 [hep-th/0302021] [SPIRES].

[48] C.P. Boyer and K. Galicki, Sasakian geometry, holonomy, and supersymmetry, math/0703231 [SPIRES].

[49] J.-B. Butruille, Homogeneous nearly Kähler manifolds, math/0612655 [SPIRES].

[50] J. Wolf, Spaces of constant curvature, McGraw-Hill, New York U.S.A. (1967).

[51] J.A. Wolf and A. Gray, Homogeneous spaces defined by Lie group automorphisms. I, J. Diff. Geom. 2 (1968) 77.

[52] T. Friedrich and S. Ivanov, Parallel spinors and connections with skew-symmetric torsion in string theory, Asian J. Math. 6 (2002) 303 [math/0102142].

[53] M. Fernández, S. Ivanov, V. Muñoz and L. Ugarte, Nearly hypo structures and compact Nearly Kähler 6-manifolds with conical singularities, math/0602160 [SPIRES].

[54] F.M. Cabrera, SU(3)-Structures on hypersurfaces of manifolds with $G_{2}$-structure, Monatsh. Math. 248 (2006) 29 [math/0410610].

[55] T. Friedrich, Nearly Kaehler and nearly parallel $G_{2}$-structures on spheres, math/0509146. 